\title{
Research on Collaborative Selection of Complex Product Systems
}

\author{
Sai Zhang* \\ School of Management \\ Henan University of Science and Technology \\ LuoYang, HeNan, China \\ Zhangs2020@sina.com
}

\author{
Fengke Wang \\ School of Management \\ Henan University of Science and Technology \\ LuoYang, HeNan, China
}

\begin{abstract}
Whether a complex product system integrator can reasonably choose a collaborator is one of the key factors for the success of a complex product system $R \& D$ project. The study aims to provide a scientific basis for the selection of suitable collaborators for complex product system integrators, which offers a practical guide for the smooth development of complex product system $R \&$ D projects. Firstly, this work summarized the factors affecting the selection of collaborators based on the characteristics of complex product systems. Then, an evaluation index system model for collaborators of complex product systems based on AHP and gray correlation methods was built. Finally, an example analysis identified the feasibility of this method. The evaluation process is simple and intuitive, and the results are scientific and effective.
\end{abstract}

Keywords-complex product system; collaborative quotient selection; grey relation degree; AHP; comprehensive evaluation

\section{INTRODUCTION}

The 19th National Congress of the Communist Party of China emphasized the promotion of key core technologies and major technological infrastructures, and clearly pointed out that it was necessary to optimize the innovation ecology and mobilize the enthusiasm of various innovation entities. In the 2018 "Government Work Report", Premier Li Keqiang proposed a new idea for the development of China's manufacturing industry, which is to create the "Made in China 2025" demonstration zone and accelerate to construct a strong country[1]. Given that, it can be seen that the manufacturing industry of China undergoes the transformation and strives to climb from the low-end to the high-end of the value chain from traditional industries to strategic emerging industries with a complex product system.

The concept of a complex product system (CoPS) is originated from the US military system and evolved from the concept of a large technology system [2]. A complex product system is a large-scale product, system, and infrastructure. It features high R\&D costs, large scale, high technical content and small batch production. [3]. There are many systems, such as large computers, large-scale communication systems, aerospace systems, power network control systems, high-speed rail, as well as semiconductor production lines.

Stakeholders in complex product systems mainly contain system integrators, collaborators, suppliers, users, government agencies, and industry regulatory associations. As a large-scale capital and technology-intensive innovation product, complex product systems possess high $R \& D$ costs, large scale, complicated systems, long project cycles, and high failure rates [4]. Therefore, it is challenging for system integrators to have all resources that are required for a complex product system innovation. It needs to form a technical alliance with relevant research institutions, and separate tasks into relatively independent modules based on technical and functional requirements. Then, it requires to subcontract them to relevant research institutions with competence [5]. This study refers to enterprises, research institutes, and universities involved in relevant research institutions as collaborators.

The data prove that the failure rate of cooperative technology innovation is as high as $50 \%$ 60\%, which is mainly ascribed to inappropriate partner selection [6]. Hence, whether complex product system integrators can choose the right collaborators is a key factor in the success of complex product system R\&D projects. Therefore, this study is about choosing the right technology alliance partner-collaborator for system integrators. Currently, there are rare reports about the choice of CoPS collaborators. Most reports only provide simple evaluations of partners based on factors such as time, cost and technology. Moreover, a comprehensive evaluation model of collaborative business selection indicators based on features of complex product systems is rarely established. To this end, this study combines the characteristics of complex product systems and the principle of partner selection to construct a comprehensive product system collaborative evaluation index system. Moreover, a gray fuzzy evaluation method combining an analytic hierarchy process with a grey correlation degree is adopted, which aims to construct a selection evaluation model for the complex product system collaborators. This study can provide a theoretical and practical basis for the system integrator to choose the appropriate collaborator.

\section{CONSTRUCTION OF GREy FuZZY COMPREHENSIVE EVALUATION MODEL}

\section{A. Principles for the selection of complex product system partners}

For complex product systems, as its technology alliance, the appropriateness of the choice of the collaborator directly determines whether the complex product system research and development project can be successfully implemented. At the beginning of establishing the alliance, the complex product 
strategic goals and organizational culture will facilitate their

system will invest ample manpower, along with material and financial resources. The alliance crisis caused by inappropriately selecting collaborators may result in immeasurable losses [6]. Therefore, careful selection of its technical support partners can effectively reduce the risk of complex product system projects. To make the evaluation index system of complex product system partners more scientific and effective, these principles should be followed:

1) The principle of complementarity of innovation and technical ability

The development of complex product systems requires extensive knowledge and skill participation, and the integration of novel technologies into development and production. There still exists the uneven allocation of technological innovation capabilities and resource levels between different enterprises or institutions. The formation of technology alliances can reconfigure technological innovation capabilities and resources to form complementary effects. The integration and exchange of core technologies of collaborative innovation partners can effectively decrease the project development cycle and enhance the success rate of complex product system projects. Therefore, the core basis for the selection of evaluation indicators for complex product system partners relies on whether the partners have technical capabilities and complementary resources.

2) The principle of combining "project orientation" and "relationship orientation"

The "task-oriented" factor emphasizes the high-tech and innovative capabilities that partners should possess when a research and development entity of a complex product system completes certain project objectives. The "relationship-oriented" factor underlines the compatibility of strategic goals, organizational culture, etc., among partners. It is extremely crucial for project leader system integrators to communicate and coordinate with various stakeholders during a long-term innovation process of complex product systems. Compatible communication. Based on the "task-oriented" and "relationship-oriented" factors, we can select the right partners to ensure the stable development of complex product system cooperation and innovation projects.

\section{3) "Reciprocal symbiosis" principle}

A high-tech enterprise technology alliance is a typical interest symbiosis. Its formation, development and cultivation are very similar to the evolution process of living organisms [7] Essentially, the complex product system refers to a technology innovation organization led by system integrators and a technology alliance with collaborators, which aims to meet the demands of users. When the symbiotic enterprise chooses other candidate symbiotic objects, the first principle of its choice is to enhance its own functions. The ability and compatibility (low matching cost) are preferred.

\section{B. Selection of evaluation indicators for complex product systems}

According to the above principles, this study constructs an initial evaluation index system for complex product system partners, including the target layer, criterion layer and indicator layer.

In order to ensure the rationality of the indicator system, the expert survey method is applied to analyze and screen the initial evaluation index system in this study. The criteria layer 4 indicators include technical and resource complementarity, cooperation compatibility, cooperation risk, and financial status. 11 indicators at the indicator level contain proprietary technology capabilities and resources, research and development capabilities, organizational management capabilities, manufacturing capabilities, organizational culture compatibility, strategic objectives compatibility, self-flexibility, profitability, asset-liability, as well as cooperation history and exit difficulty (as shown in TABLE I).

TABLE I. CHOOSE INITIAL EVALUATION INDEX SYSTEM

\begin{tabular}{|c|c|c|c|}
\hline Target layer & Criteria layer & Indicator layer & Indicator layer interpretation \\
\hline \multirow{17}{*}{$\begin{array}{c}\text { Complex product system partners } \\
\text { choose initial evaluation index } \\
\text { system }\end{array}$} & \multirow{9}{*}{$\begin{array}{l}\text { Technology and } \\
\text { resources } \\
\text { Complementarity } \\
\text { /X1 }\end{array}$} & \multirow{2}{*}{$\begin{array}{l}\text { Professional technical skills and } \\
\text { resources/X11 }\end{array}$} & Technology and resource differentiation /X111 \\
\hline & & & Technology and resource relevance/X112 \\
\hline & & \multirow{3}{*}{ R \& D capabilities/X12 } & $\mathrm{R} \& \mathrm{D}$ ratio/X121 \\
\hline & & & Developer ratio/X122 \\
\hline & & & Technical patent grant/X123 \\
\hline & & \multirow[b]{2}{*}{ Manufacturing capacity/X13 } & Relevant equipment advanced level/X131 \\
\hline & & & $\begin{array}{c}\text { Production and manufacturing personnel } \\
\text { quality/X132 }\end{array}$ \\
\hline & & \multirow{2}{*}{$\begin{array}{l}\text { Organizational management ability } \\
\text { /X14 }\end{array}$} & Leading customer contact/X141 \\
\hline & & & Communication and coordination/X142 \\
\hline & \multirow{3}{*}{$\begin{array}{l}\text { Cooperative } \\
\text { compatibility } \\
\text { /X2 }\end{array}$} & Organizational culture compatibility /X21 & Organizational culture compatibility/X211 \\
\hline & & Strategic goal compatibility/X22 & Consistency of strategic objectives/X221 \\
\hline & & Self-flexibility/X23 & Cross-organizational participation/X231 \\
\hline & \multirow{2}{*}{$\begin{array}{l}\text { Financial status } \\
\text { /X3 }\end{array}$} & Profitability/X31 & Operating net profit margin/X311 \\
\hline & & Asset-liability/X32 & Assets and liabilities/X321 \\
\hline & \multirow{3}{*}{$\begin{array}{l}\text { Cooperation risk } \\
\text { /X4 }\end{array}$} & Cooperation history/X41 & Organizational damage rate/X411 \\
\hline & & \multirow{2}{*}{ Exit difficulty/X42 } & Expected input/X421 \\
\hline & & & Reputation pays attention to the situation/X422 \\
\hline
\end{tabular}


C. Grey Fuzzy Comprehensive Evaluation Model for Complex Product System Collaborators

The index system for collaborative evaluation of complex product systems features multi-level, multi-factor and uncertainty. In order to ensure a scientific and effective evaluation, this study selects a fuzzy comprehensive evaluation method to evaluate and select candidate collaborators. The analytic hierarchy process is used to determine the weight, and the grey correlation coefficient is performed to determine the degree of membership. The problem of subjectivity in the evaluation process is solved.

1) Weight Assignment Calculation Based on the Analytic Hierarchy Process

a) The elements in the factor set $X=(x 1, x 2, \ldots, x m)$ are compared in pairs. The importance contains 1 to 9 scales, and the ratio of the $i$-th element can be obtained as ai1, ai2.... aij. Given that, a judgment matrix is constructed.

b) Calculate the product Mi of each row element of the judgment matrix, $\mathrm{Mi}=a i 1^{*}$ ai ${ }^{*} \ldots *$ aij ; calculate the $n t h$ root of $M i$, and obtain $W i=\sqrt[n]{W i}$;

c) The normalization process of $\mathrm{Wi}$ is obtained, and $W_{O}=W i / \sum_{i=1}^{n} W i W_{O}=(W 1, W 2, \cdots W n) T$ is the feature vector of the judgment matrix, namely, the weight of each indicator.

2) The solution of Membership Degree Based on Grey Correlation Degree

a) Determine the optimal set of indicators for the indicator layer

In this study, based on the grey fuzzy comprehensive evaluation analysis, $\mathrm{U}^{*}=\left\{\mathrm{u} 1^{*}, \mathrm{u} 2^{*}, \ldots, \mathrm{um}^{*}\right\}$ is set as the optimal index set. The optimal values in this indicator set are the best value among many candidate solutions. Thus, the initial matrix E can be constructed as follows.

$$
\mathrm{E}=\left[\begin{array}{cccc}
u_{1}^{*} & u_{2}^{*} & \cdots & u_{m} * \\
u_{11} & u_{12} & \cdots & u_{1 m} \\
u_{21} & u_{22} & \cdots & u_{23} \\
\vdots & \vdots & & \vdots \\
u_{n 1} & u_{n 2} & \cdots & u_{n m}
\end{array}\right]
$$

b) Dimensionless processing of indicator values

Considering that indicators in the evaluation index system possess various dimensions and cannot be directly compared, the original data should be dimensionless. Assuming that the minimum value of the i-th factor indicator ui is uimin, and the maximum value is uimax. Then:

$$
C=\frac{u_{j i}-u_{i}^{\min }}{u_{i}^{\max }-u_{i}^{\min }}(i=1,2, \cdots, m ; j=1,2, \cdots, n)
$$

\section{c) Calculate the grey correlation coefficient}

After the dimensionless processing, the optimal index set $\mathrm{C}^{*}=\left(\mathrm{C} 1^{*}, \mathrm{C} 2^{*}, \cdots, \mathrm{Cm}^{*}\right)$ is used as the reference data column; the index value $\mathrm{Cj}$ of each candidate object $(\mathrm{j}=1,2, \ldots, \mathrm{m})$, $\mathrm{Cj}=(\mathrm{Cj} 1, \mathrm{Cj} 2, \ldots, \mathrm{Cjm})$ as the compared series; the correlation coefficient of the jth candidate object with the optimal index ui* under the action of the index uji of the $i$-th factor ui is $\eta j(i)$ $(\mathrm{i}=1,2, \ldots, \mathrm{m} ; \mathrm{j}=1,2, \ldots \mathrm{n})$.

$$
\begin{gathered}
\eta_{j i}=\frac{\Delta C_{\min }+\sigma \Delta C_{\max }}{\Delta C_{j i}+\sigma \Delta C_{\max }} \\
\Delta C_{\min }=\frac{\min }{j} * \frac{\min }{i} *\left|c i^{*}-c j i\right| ; \\
\Delta C_{\max }=\frac{\max }{j} * \frac{\max }{i} *\left|c i^{*}-c j i\right| ; \\
\Delta C_{j i}=\left|c j i-c i^{*}\right| ; \sigma \in[0,1] .
\end{gathered}
$$

The formula refers to the degree to which the ideal solution that the scheme may achieve in terms of the indicator cij after all possible influences of the cji on the corresponding criterion layer ci, namely, the gray fuzzy membership. Based on this, the relative gray fuzzy membership matrix of each index can be achieved.

$$
\mathrm{R}=\left[\begin{array}{cccc}
\eta 11 & \eta 12 & \cdots & \eta 1 m \\
\eta 21 & \eta 22 & \cdots & \eta 2 m \\
\cdots & \ldots & \ldots & \cdots \\
\eta n 1 & \eta n 2 & \cdots & \eta n m
\end{array}\right]
$$

d) Fuzzy Evaluation and Analysis of Its Results

The weight vector of $\mathrm{m}$ evaluation indicators relative to the total target is $W=(w 1, w 2, \cdots, w m)$ T. An association vector $B$ is constructed by the weighted association degree of each supplier $\mathrm{Xi}$ and the relative optimal solution $\mathrm{Xo}, \mathrm{B}=\eta^{*} \mathrm{~W}$,

Then, the candidate collaborators according to the size of $\mathrm{B}$ is classified. A larger $\mathrm{B}$ results in a closer supplier $\mathrm{X}$ i to the optimal scheme Xo. Therefore, when $\mathrm{Bi}=\max (\mathrm{B} 1, \mathrm{~B} 2, \ldots$, $\mathrm{Bm})$, the supplier $\mathrm{X} \mathrm{i}$ is the optimal supplier.

\section{CASE ANALYSIS}

An enterprise $E$ contracted a complex product system project of "waste tire solid heat carrier full-cycle high efficient dry distillation cracking comprehensive utilization technology". The project mainly investigates the recycling and pollution synergy control theory system. This is adapted to China's solid waste characteristics, which is based on. the advantages of each technology participating entity. And it belongs to a typical complex product system. The project contains four modules. This example is intended to select a reasonable and efficient subcontractor for system integrators, which facilitates to complete the migration of harmful elements in the waste tire cracking process. The factors of the evaluation indicators are selected as all factors, as shown in Table I. There are three candidate companies, namely, $\mathrm{D}, \mathrm{E}$, and $\mathrm{H}$. 
A. Weight Assignment Calculation Based on the Analytic Hierarchy Process

TABLE II. THE WEIGHT OF THE FIRST-LEVEL INDICATOR X AND ITS CONSISTENCY TEST

\begin{tabular}{c|cccc|c|c|c|c}
\hline$X$ & $X 1$ & $X 2$ & $X 3$ & $X 4$ & Wi & Wi0 & & \\
\hline$X 1$ & 1 & 3 & 5 & 2 & 2.340 & 0.470 & 4.073 & $\lambda \max \approx 4.053$ \\
$X 2$ & $1 / 3$ & 1 & 3 & $2 / 3$ & 0.904 & 0.182 & 4.018 & C.I. $=0.018$ \\
$X 3$ & $1 / 5$ & $1 / 3$ & 1 & $1 / 5$ & 0.340 & 0.068 & 4.083 & C.R. $=$ C.I. $/$ R.I. $=0.02<0.1$ \\
$X 4$ & $1 / 2$ & $3 / 2$ & 5 & 1 & 1.392 & 0.280 & 4.036 & \\
\hline \multicolumn{10}{|c|}{} & \\
\hline
\end{tabular}

TABLE III. THE WEIGHT OF THE SECOND-LEVEL INDICATOR $\mathrm{X}_{1}$ AND ITS CONSISTENCY TEST

\begin{tabular}{c|cccc|c|c|c|c}
\hline$X$ & $X 11$ & $X 12$ & $X 13$ & $X 14$ & Wi & Wi0 & & \\
\hline$X 11$ & 1 & 1 & 5 & 6 & 2.340 & 0.423 & 4.001 & $\lambda \max \approx 4.000$ \\
$X 12$ & 1 & 1 & 5 & 6 & 2.340 & 0.423 & 4.001 & C.I. $=0$ \\
$X 13$ & $1 / 5$ & $1 / 5$ & 1 & $6 / 5$ & 0.468 & 0.084 & 4 & C.R. $=$ C.I./ R.I. $=0<0.1$ \\
$X 14$ & $1 / 6$ & $1 / 6$ & $5 / 6$ & 1 & 0.390 & 0.070 & 4 & \\
\hline \multicolumn{7}{|c|}{} \\
\hline
\end{tabular}

Similarly, the second-level indicator weight vector is: $w 3=(0.75,0.25), w 4=(0.333,0.667)$. $w 1=(0.423,0.423,0.084,0.07), w 2=(0.158,0.158,0.684)$,

TABLE IV. THE WEIGHT OF THE THIRD-LEVEL INDICATOR $\mathrm{X}_{11}$ AND ITS CONSISTENCY TEST

\begin{tabular}{c|cc|c|c|c|c}
\hline $\mathrm{X}$ & $X 111$ & $\mathrm{X112}$ & Wi & Wi0 & & \\
\hline$X 111$ & 1 & 2 & 1.414 & 0.667 & 2 & $\lambda \max =2$ \\
$X 112$ & $1 / 2$ & 1 & 0.707 & 0.333 & 2 & C.I. $=0$ \\
\hline \multicolumn{7}{c}{2.121} \\
\hline
\end{tabular}

Similarly, the weight vector of each level three indicator can be expressed as $w 11=(0.667,0.333)$,

$w 12=(0.158,0.158,0.684), w 13=(0.5,0.5), w 14=(0.5,0.5)$, $w 41=(0.75,0.25)$.

The weight vector of the primary indicator is $w=(0.470,0.182,0.068,0.280)$

The weight of each specific indicator relative to the target layer can be achieved by multiplying each indicator layer weight by the criterion layer weight:
$W=\{0.133,0.066,0.031,0.031,0.136,0.020,0.020,0.016$, $0.016,0.029,0.029,0.124,0.051,0.017,0.193,0.140,0.047\}$

\section{B. A solution of Membership Degree Based on Grey Correlation Degree}

For the three candidate enterprises of D, H, E, the original data are displayed in Table $\mathrm{V}$.

1) According to Table 6, the optimal reference series of indicators can be obtained

$$
U^{*}=\{0.9,0.9,0.5,0.289,1825,0.9,0.9,1,0.9,1,0.9,1,0.9,0.9 \text {, }
$$$$
0.2,0.19,0.9\}
$$

TABLE V. EVALUATION INDICATORS OF THREE CANDIDATE COMPANIES' RAW DATA

\begin{tabular}{|c|c|c|c|c|c|c|c|c|c|c|c|c|c|c|c|c|c|}
\hline & $X 111$ & X112 & $X 121$ & $X 122$ & $X 123$ & $X 131$ & X132 & $X 141$ & $X 142$ & $X 211$ & $X 221$ & X231 & X311 & X321 & $X 411$ & $X 421$ & $X 422$ \\
\hline D & 0.9 & 0.9 & 0.038 & 0.13 & 1020 & 0.8 & 0.7 & 0.9 & 0.7 & 1 & 0.9 & 0.7 & 0.9 & 0.7 & 0.1 & 0.19 & 0.9 \\
\hline $\boldsymbol{H}$ & 0.7 & 0.8 & 0.05 & 0.289 & 1284 & 0.9 & 0.9 & 0.9 & 0.9 & 0.9 & 0.7 & 0.9 & 0.7 & 0.9 & 0.2 & 0.15 & 0.9 \\
\hline$E$ & 0.9 & 0.7 & 0.05 & 0.272 & 1825 & 0.7 & 0.7 & 1 & 0.8 & 0.9 & 0.7 & 1 & 0.9 & 0.7 & 0.1 & 0.09 & 0.7 \\
\hline
\end{tabular}

2) The dimension value of the index value is obtained, and the matrix $C$ can be derived. The values are shown in Table VI.
3) Calculate the correlation coefficient of each enterprise according to the matrix $C$, and obtain the correlation coefficient matrix, namely, the gray fuzzy membership matrix

TABLE VI. STANDARDIZATION OF THREE CANDIDATE ENTERPRISE EVALUATION INDICATORS

\begin{tabular}{|c|c|c|c|c|c|c|c|c|c|c|c|c|c|c|c|c|c|}
\hline & $\begin{array}{llll}X & 111 \\
\end{array}$ & $\begin{array}{llll}X & 11\end{array}$ & $\begin{array}{lll}X 121 \\
\end{array}$ & $\begin{array}{lll}X 122 \\
\end{array}$ & $\begin{array}{llll}X & 123 \\
\end{array}$ & $\begin{array}{llll}X & 131 \\
\end{array}$ & $\begin{array}{llll}X & 1 & 3 & 2 \\
\end{array}$ & $X 141$ & $X 142$ & $X 211$ & $\begin{array}{lll}X & 221 \\
\end{array}$ & \begin{tabular}{llll|} 
& 2 & 3 & 1
\end{tabular} & $X 311$ & X 321 & $X 411$ & $X 421$ & $X 422$ \\
\hline$D$ & 1 & 1 & 0 & 0 & 0 & 0.5 & 0 & 0 & 0 & 1 & 1 & 0 & 1 & 0 & 0 & 1 & 1 \\
\hline $\boldsymbol{H}$ & 0 & 0.5 & 1 & 1 & 0.328 & 1 & 1 & 0 & 1 & 0 & 0 & 0.667 & 0 & 1 & 1 & 0.6 & 1 \\
\hline $\boldsymbol{E}$ & 1 & 0 & 1 & 0.893 & 1 & 0 & 0 & 1 & 0.5 & 0 & 0 & 1 & 1 & 0 & 0 & 0 & 0 \\
\hline
\end{tabular}




$$
\mathrm{R}=\left[\begin{array}{ccccccccccccccccc}
1 & 1 & 0.333 & 0.333 & 0.333 & 0.5 & 0.333 & 0.333 & 0.333 & 1 & 1 & 0.333 & 1 & 0.333 & 0.333 & 1 & 1 \\
0.333 & 0.5 & 1 & 1 & 0.427 & 1 & 1 & 0.333 & 1 & 0.333 & 0.333 & 0.6 & 0.333 & 1 & 1 & 0.556 & 1 \\
1 & 0.333 & 1 & 0.824 & 1 & 0.333 & 0.333 & 1 & 0.5 & 0.333 & 0.333 & 1 & 1 & 0.333 & 0.333 & 0.333 & 0.333
\end{array}\right]
$$

4) Calculate the correlation vector $B$ according to $B=\eta \times W$, $B=(0.669,0.704,0.759)$. Hence, $B E=\max (B D, B H, B E)$, and the collaborator $E$ is the first choice among the candidate companies.

\section{CONCLUSION}

In fact, complex product system innovation can remarkably reflect the comprehensive national strength and technological competitiveness of a country, which renders this country a proactive position in the current international competition dominated by technology and knowledge. However, due to the large scale of complex product systems, complex systems, long project cycles, and high failure rates, system integrators generally guarantee the successful completion of complex product system projects by selecting high-tech and scientifically effective collaborators. In this study, the integration of the analytic hierarchy process and grey correlation degree is applied to select collaborators and effectively binder the expert wisdom and objective data. Finally, by analyzing examples, it has been proved that the algorithm is feasible; the evaluation process is simple and intuitive; the evaluation results are scientific and effective. The results of this study have high practical significance.

\section{REFERENCES}

[1] Li Keqiang. 2019 State Council Government Work Report [EB/OL].http://www.gov.cn/premier/2019-03/16/content_5374314.htm.

[2] Hobday, M. Product complexity, innovation and industrial organization, 26rd ed., vol. 2. Research Policy, 1998, pp.689 - 710.

[3] Hansen, K.L. Rush, H. Hotspots in complex product systems: Emerging issues in innovation management, 18rd ed., vol. 9. Technovation, 1998, pp.555-561.

[4] DEDEHAYIR O, NOKELAINEN T, MÄKINEN S J. Disruptive innovations in complex product systems industries: A case study,33rd ed., vol. C. Journal of Engineering \& Technology Management, 2014, pp.174-192.

[5] Shou Yongyi, Song Yijiang. Partner Selection and Optimization of Complex Product System R\&D Projects, 10rd ed, Research Management, 2014, pp.144-149. (In Chinese)

[6] You Daming, Huang Yizi. The Choice and Evaluation of Breakthrough Technology Innovation Partners, 32nd ed., vol.3.System Engineering, 2014, pp.99-103. (In Chinese)

[7] Xue Weixian, Zhang Juan. Research on Partner Selection for Mutualism and Symbiosis in High-tech Enterprise Technology Alliance, 22nd ed, vol.1. Research and Development Management, 2010, pp.82-89 + 113 . (In Chinese). 